\title{
Social Experience Interacts with Serotonin to Affect Functional Connectivity in the Social Behavior Network following Playback of Social Vocalizations in Mice
}

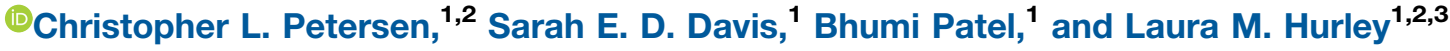

https://doi.org/10.1523/ENEURO.0247-20.2021

${ }^{1}$ Department of Biology, Indiana University Bloomington, Bloomington, IN $47405,{ }^{2}$ Center for the Integrative Study of Animal Behavior, Indiana University Bloomington, Bloomington, IN 47405, and ${ }^{3}$ Department of Neuroscience, Indiana University Bloomington, Bloomington, IN 47406

\begin{abstract}
Past social experience affects the circuitry responsible for producing and interpreting current behaviors. The social behavior network (SBN) is a candidate neural ensemble to investigate the consequences of early-life social isolation. The SBN interprets and produces social behaviors, such as vocalizations, through coordinated patterns of activity (functional connectivity) between its multiple nuclei. However, the SBN is relatively unexplored with respect to murine vocal processing. The serotonergic system is sensitive to past experience and innervates many nodes of the SBN; therefore, we tested whether serotonin signaling interacts with social experience to affect patterns of immediate early gene (IEG; cFos) induction in the male SBN following playback of social vocalizations. Male mice were separated into either social housing of three mice per cage or into isolated housing at 18-24d postnatal. After 28-30 d in housing treatment, mice were parsed into one of three drug treatment groups: control, fenfluramine (FEN; increases available serotonin), or pCPA (depletes available serotonin) and exposed to a 60-min playback of female broadband vocalizations (BBVs). FEN generally increased the number of cFos-immunoreactive (-ir) neurons within the SBN, but effects were more pronounced in socially isolated mice. Despite a generalized increase in cFos immunoreactivity, isolated mice had reduced functional connectivity, clustering, and modularity compared with socially reared mice. These results are analogous to observations of functional dysconnectivity in persons with psychopathologies and suggests that early-life social isolation modulates serotonergic regulation of social networks.
\end{abstract}

Key words: auditory; functional connectivity; serotonin; social behavior network; social isolation; vocal processing

\section{Significance Statement}

Social isolation and serotonergic signaling each modulate neural functions independently of each other. It is unknown whether these factors interact to affect activity at the level of individual nuclei, and/or functional networks. Using a vocal playback paradigm, we find that acutely increasing or systemically depleting available serotonin increased cFos-immunoreactive (-ir) neurons in the social behavior network (SBN) of mice raised in social isolation compared with their socially reared counterparts. We show for the first time that mice raised in social isolation have reduced functional connectivity in the SBN relative to socially reared mice. Importantly, network perturbations were not resolved by drug treatment in isolated mice suggesting that social experience is necessary to facilitate functional relationships in the SBN.

Received June 9, 2020; accepted February 15, 2021; First published March 3, 2021.

The authors declare no competing financial interests.
Author contributions: C.L.P. and L.M.H. designed research; C.L.P., S.E.D.D., B.P., and L.M.H. performed research; C.L.P. and L.M.H. analyzed data; C.L.P. wrote the paper. 


\section{Introduction}

The importance of social rearing has been evident since the 1960s and the now-controversial "Harlow monkey experiments," which demonstrated that early-life social isolation deprives macaques of the experiences necessary to develop into functional adults (Harlow et al., 1965). The ability to amicably behave and communicate with conspecifics is important to social cohesion, which in turn affects individual fitness and psychological wellness (Heim and Nemeroff, 2001; Bailey and Moore, 2018). Neural systems have evolved to support social cohesion (Goodson, 2013; Matthews and Tye, 2019) such that social interactions may carry positive valence (Goodson and Wang, 2006), and be reinforced by the brain's reward circuitry (Dölen et al., 2013). Early-life social immersion or deprivation may shape the circuits responsible for the appropriate expression of social behaviors.

One such circuit is the vertebrate social behavior network (SBN). This includes the lateral septum (LS); bed nucleus of the stria terminalis (BNST); medial preoptic area (mPOA); paraventricular (PVN), anterior $(\mathrm{AH})$, and ventromedial $(\mathrm{VMH})$ hypothalamic nuclei; the ventral tegmental area (VTA); and periaqueductal gray (PAG; Newman, 1999; Goodson, 2005; Goodson and Kingsbury, 2013). Rather than an exhaustive list of regions that facilitate social processes (Rogers-Carter and Christianson, 2019), the SBN is an evolutionarily conserved suite of nuclei responsible for interpreting and generating responses to social stimuli (Goodson, 2005; O'Connell and Hofmann, 2012). SBN nuclei or "nodes" are differentially engaged during specific behaviors (Lin et al., 2011; Lee et al., 2014; Decot et al., 2017; McHenry et al., 2017; Kohl et al., 2018; Tschida et al., 2019); however, coordinated patterns of activity or functional connectivity (Friston, 2011) across the SBN contribute to variation in behavioral output (Goodson, 2005; Goodson and Kabelik, 2009).

Since the original proposal of the SBN (Newman, 1999), analytical tools have been developed to quantitatively describe functional anatomic networks (Sporns, 2010; Fornito et al., 2016). For example, metrics such as density measure functional connectivity in a given network, whereas the clustering coefficient is an indicator of "small-world" networks which display increased efficacy of communication among

This work was supported by the National Institute of Health Common Themes in Reproductive Diversity Training Grant 5T32HD049336-12, the Indiana University College of Arts and Sciences, and the Center for the Integrative Study in Animal Behavior (C.L.P) and the National Science Foundation Grant IOS 1456298 (L.M.H.).

Acknowledgements: We thank Jim Powers in the Indiana University Light Microscopy Imaging Center (LMIC) for technical support, Richard Betzel for network advice, Kelly Ronald, and Anna Ingebretson for comments on the original manuscript, and members of the Hurley Lab for helpful discussions. This publication is inspired by and dedicated to the memory of James L. Goodson (1965-2014).

Correspondence should be addressed to Christopher L. Petersen at chlpete@umn.edu.

https://doi.org/10.1523/ENEURO.0247-20.2021

Copyright (C) 2021 Petersen et al.

This is an open-access article distributed under the terms of the Creative Commons Attribution 4.0 International license, which permits unrestricted use, distribution and reproduction in any medium provided that the original work is properly attributed. regions (Watts and Strogatz, 1998). Community structure/ modularity calculates the degree to which nodes assemble into functionally similar clusters (Newman and Girvan, 2004). Functional networks are disrupted in human psychopathologies (Bullmore et al., 1997; van den Heuvel and Sporns, 2019), emphasizing the importance of investigating network-level features in rodent translational models (Van den Heuvel et al., 2016). Network-based analyses in non-traditional model systems describe changes in SBN functional connectivity following presentation of socially salient vocal stimuli (Hoke et al., 2005; Ghahramani et al., 2018); however, no such studies exist in laboratory mice.

Murine vocalizations are a source of context-dependent information during social interactions (Hanson and Hurley, 2012; Finton et al., 2017; Warren et al., 2018, 2020; Sangiamo et al., 2020). Vocal processing relies on auditory circuitry as well as functionally diverse nuclei such as the SBN. For example, receivers must extract the physical characteristics of vocal signals (e.g., frequency, duration, amplitude, etc.) and interpret them in light of their own experiences and current conditions (Petersen and Hurley, 2017). Investigating whether social isolation disrupts vocal processing in circuits such as the SBN will be important in understanding the mechanisms underlying aberrant behavior in mouse models of communicative and affective disorders (Portfors and Perkel, 2014).

Serotonergic signaling is sensitive to social isolation: socially isolated mice downregulate 5-HT receptor expression in hypothalamic nodes of the SBN (Schiller et al., 2003; Bibancos et al., 2007). As anatomically distinct regions of the dorsal raphe nucleus send serotonergic projections to the SBN (Schwarz et al., 2015; Muzerelle et al., 2016; Beier et al., 2019), serotonin may modulate SBN activity in accordance with an animal's internal state and changes in the external environment (Muzerelle et al., 2016; Niederkofler et al., 2016; Ren et al., 2018). Broadly activating serotonergic pathways affects neural activity markers across a distributed suite of nuclei including the SBN (Giorgi et al., 2017; Grandjean et al., 2019); however, it remains unknown whether social experience interacts with serotonin signaling to affect activity-dependent measures and network-level metrics such as functional connectivity.

We use immediate early gene (IEG) mapping to test the hypothesis that serotonin signaling interacts with social experience to affect patterns of cFos-immunoreactive (-ir) neurons in the SBN of male mice following presentation of female broadband vocalizations (BBVs). Increasing available serotonin increased the IEG response in several SBN nodes. This effect was more prominent in socially isolated mice regardless of drug treatment. Despite increases in cFos-ir neurons, network analyses reveal fewer functional relationships within the $\mathrm{SBN}$ of socially isolated mice.

\section{Materials and Methods}

\section{Animal information}

The Indiana University, Bloomington Institutional Animal Care and Use Committee (protocol \#15-021) approved all of the following experiments. Individual cohorts 
A Arrived from

B Separated into social C

C 28 - 30

D 3 days before playback

E Day of $\underset{\substack{\text { Playback } \\ \text { setup }}}{\text { Plup }}$ Jackson Labs or isolated housing days
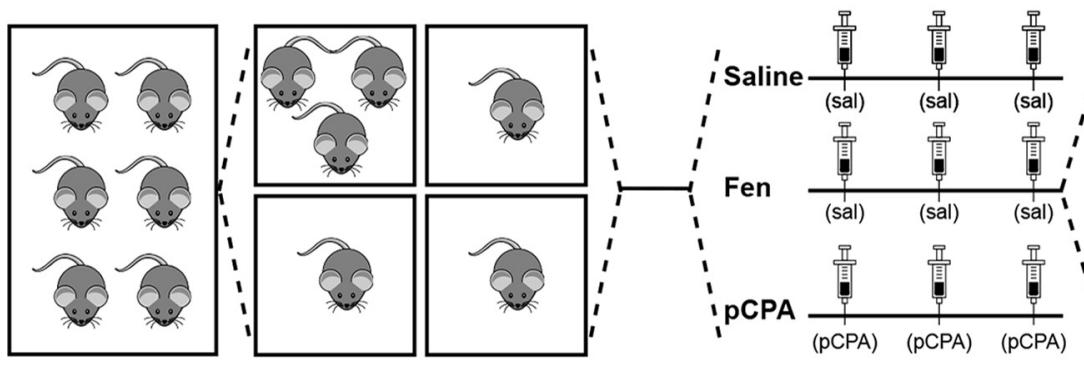
playback
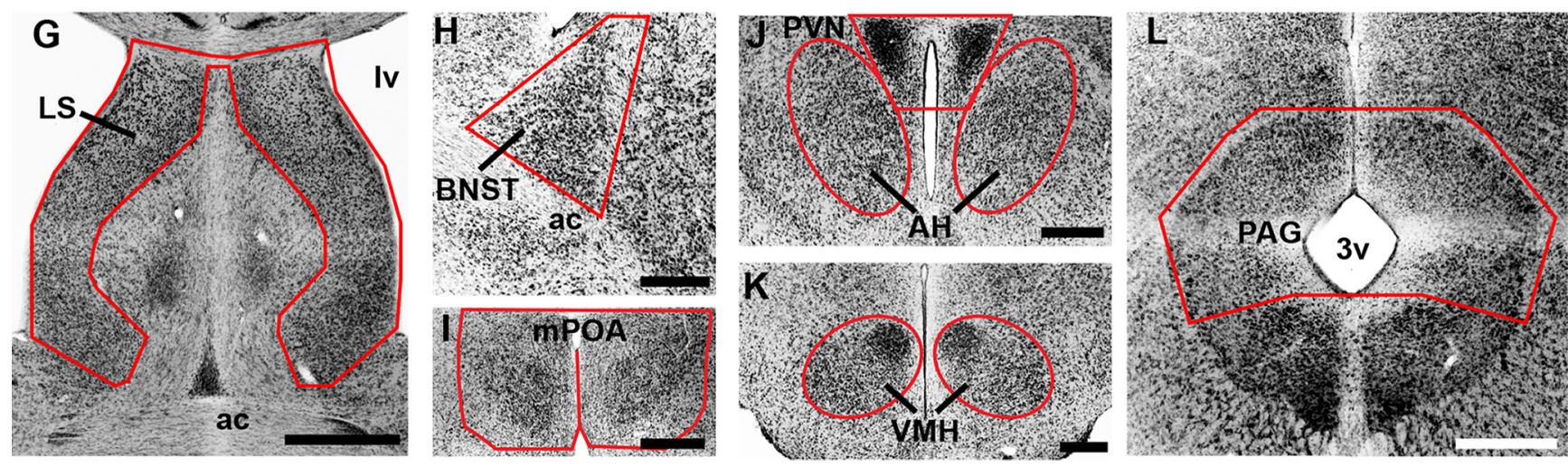

Figure 1. Experimental design: playback paradigm and neuroanatomy. $\boldsymbol{A}$, Male CBA/J mice arrived from The Jackson Laboratory at 18-24 d postnatal and were immediately separated into social (three per cage) or isolated (one per cage) housing (B). $\boldsymbol{C}, \mathrm{Mice}$ remained in their respective housing conditions for 28-30 d. $\boldsymbol{D}$, SAL and FEN mice received SAL injections for $3 \mathrm{~d}$ before playback; mice in the pCPA group received pCPA injections on these days. $\boldsymbol{E}$, Forty-five minutes before playback trials, mice in the SAL and pCPA group received SAL injections, whereas FEN mice received FEN. $\boldsymbol{F}$, Playback trials were 60 min and consisted of $14-15$ bursts of five female BBVs. G-L, Representative inverse fluorescent $10 \times$ photomicrographs showing seven nodes of the SBN: LS (G), BNST $(\boldsymbol{H})$, mPOA $(\boldsymbol{I}), \mathrm{PVN}$ and $\mathrm{AH}(\boldsymbol{J}), \mathrm{VMH}(\boldsymbol{K})$, and PAG $(\boldsymbol{L})$. ac, anterior commissure; Iv, lateral ventricle; $3 \mathrm{v}$, third ventricle. Scale bars: $1 \mathrm{~mm}(\boldsymbol{G})$ and $500 \mu \mathrm{m}(\boldsymbol{H}-\boldsymbol{L})$.

of male CBA/J mice (Mus musculus) from different litters were shipped from The Jackson Laboratory and received at 18-24 d of age (Fig. 1A). Each cohort was assigned to 1 of three pharmacological treatment groups: saline (SAL; control), fenfluramine (FEN), or pCPA (see pharmacological details below). Upon arrival, mice were separated into either social housing of three mice per cage or into isolated housing (Fig. 1B). Mice remained in social (SOC) or isolated (ISO) conditions on a 14/10 h light/dark cycle with ad libitum access to food and water and weekly cage changes for 28-30 d before vocal playback (Fig. 1C,D). ISO mice were physically separated from conspecifics; however, all experimental animals were housed in the same room within our vivarium. While ISO mice were potentially exposed to olfactory, auditory, and/or visual stimuli from neighboring cages, similar conditions did not attenuate the effects of social isolation in other studies (Keesom et al., 2017a,b; Manouze et al., 2019).

\section{Pharmacology}

pCPA methyl ester hydrochloride (pCPA; 4-chloro-DLphenylalanine methyl ester hydrochloride; Tocris) was used to deplete systemic serotonin. pCPA is a non-reversible inhibitor of tryptophan hydroxylase $(\mathrm{TPH}$; the rate-limiting enzyme in serotonin synthesis; Koe et al., 1966). Over the course of 3-4 d, pCPA depletes serotonin in brain regions including the hippocampus, striatum, and cortex (Dailly et al., 2006). Conversely, dexfenfluramine hydrochloride (Fenluramine; (S)-N-ethyl- $\alpha$-methyl-3-(trifluoromethyl) benzeneethanamine hydrochloride; Tocris), which releases stores of vesicular serotonin and blocks its reuptake at the synapse (Davis and Faulds, 1996; Rothman and Baumann, 2002), was used to acutely increase levels of available serotonin.

FEN and PCPA were diluted in $0.9 \%$ sterile SAL within $3 \mathrm{~d}$ of use. PCPA was administered at $200 \mathrm{mg} / \mathrm{kg}$ in a volume of $5 \mathrm{ml} / \mathrm{kg}$; FEN was administered at $100 \mathrm{mg} / \mathrm{kg}$ in a volume of $5 \mathrm{ml} / \mathrm{kg}$ (Hanson and Hurley, 2016). Sterile SAL (vehicle; $10 \mathrm{ml} / \mathrm{kg}$ ) was used for all control injections. Each mouse in SOC cages received the same pharmacological treatment. Injections were administered interperitoneally following brief anesthetization with isoflurane, after which mice were returned to their home cage. Beginning $3 \mathrm{~d}$ before playback, mice were transferred from housing quarters to the experimental room where they received injections at roughly 24-h intervals in the morning. Over the course of these $3 d$, pCPA mice received PCPA injections while FEN and SAL mice received equivalent injections of sterile SAL (Fig. 1D). Following 
injections, mice remained in the experimental room for $45 \mathrm{~min}$ before being returned to the vivarium. This process was designed to habituate mice to injections and being moved between rooms to reduce non-specific cFos expression before playback trials. On the day of playback, PCPA and SAL mice were injected with sterile SAL $45 \mathrm{~min}$ before trials. FEN mice received FEN injections $45 \mathrm{~min}$ before playback (Fig. 1E). For each treatment group $n=9$ except for SOC-SAL, where $n=8$. Over the course of injections, ISO-pCPA mice lost a significant amount of weight (paired $t_{(8)}=2.82, p<0.05$, mean difference $0.43 \pm 0.15 \mathrm{~g}$ ), but there was no difference between the weights of treatment groups on the day of playback $(p=0.2)$.

\section{Playback trials}

Ninety minutes before playback trials, mice were retrieved from animal quarters and placed in a quiet room. After $45 \mathrm{~min}$, the first of three animals received an injection (as above) and was returned to its home cage (Fig. 1E). Forty-five minutes after injection, focal mice were transferred from their home cage to an identical testing cage $(12 \times 6 \times 6$ inches) with fresh bedding within in a sound attenuation chamber (Coulbourn Habitest) with an ultrasonic speaker (Ultrasonic Dynamic Speaker Vifa, Avisoft Bioacoustics) powered by an UltraSoundGate Player 116 (Avisoft Bioacoustics). Trials were monitored with a CCD video camera (30 fps) placed above the test cage, with SuperDVR software (Q-See, Digital Peripheral Solutions Inc.) and a Q-see four channel DVR PCl video capture card. Trials were $60 \mathrm{~min}$ and playback consisted of 14-15 naturalistic bursts of five female BBVs (Fig. 1F), the final number of BBVs (70-75) represented the average number of BBVs emitted during the study from which they were recorded. All mice were played back the same BBV sound file; omission of the final burst of five BBVs was counterbalanced across groups. Following trials, spectrograms of the playback were created in Avisoft, and the number of male-emitted ultrasonic vocalizations (USVs) were quantified.

\section{Playback generation}

Source BBVs were originally recorded during sociosexual interactions between male and female CBA/J mice. First, spectrograms of sociosexual interactions were generated using Avisoft SASlab Pro software; next, individual female BBVs were located, high-pass filtered to remove any potentially overlapping male USVs, and copied into a new playback audio file. We assembled naturalistic bursts of five individual BBVs and interspersed $270 \mathrm{~s}$ of silence between bursts. BBVs were calibrated by matching rms intensity of the playback (as recorded in the testing arena) to the intensity of the originally recorded vocalizations. The same condenser microphone (CM16/CMPA, Avisoft Bioacoustics) with an UltraSoundGate $116 \mathrm{Hb}$ sound card (250-kHz sample rate Avisoft Bioacoustics) was used to assess the intensities of the originally recorded vocalizations and the playback.

In naturalistic social interactions, female BBVs correlate with male-directed aggression (i.e., rejection-like behaviors), as they also emitted during mounting, BBVs are considered to be functionally ambiguous (Finton et al., 2017). Our playback file consisted of BBVs that were acquired during multiple interactions where mounting either did or did not occur. Thus, any potential structural differences in BBVs emitted during different contexts (i.e., mounting vs rejection) would not shape the overall valence of playback.

\section{Immunohistochemistry (IHC)}

Playback lasted for $60 \mathrm{~min}$, at which point focal animals remained in the sound attenuation chamber for 30 additional minutes to allow for accumulation of the cFos protein (Kovacs, 2008). Ninety minutes following the onset of playback, mice were deeply anesthetized with isoflurane and transcardially perfused with ice-cold Krebs-Henseleit solution ( $\mathrm{pH} 7.2$ ) followed by $50 \mathrm{ml}$ of $4 \%$ paraformaldehyde in phosphate buffer (PFA). Brains were extracted and postfixed overnight in PFA, transferred to $30 \%$ sucrose in PBS (pH 7.4) for $\sim 48 \mathrm{~h}$, and cut into three series of $50-\mu \mathrm{m}$ sections in the coronal plane using a freezing microtome. Sections were collected throughout the rostral-to-caudal extent of the inferior colliculus (IC; approximately bregma -5.34 thru $-4.36 \mathrm{~mm}$ ) and starting at the appearance of the median eminence (bregma $-1.94 \mathrm{~mm}$ ) through the bifurcation of the anterior commissure (AC; $\sim$ bregma $+0.38 \mathrm{~mm}$ ). Sections were stored in cryoprotectant solution at $-80^{\circ} \mathrm{C}$ until IHC. Three separate IHC runs counterbalanced across treatment groups were performed as follows.

Tissue was equilibrated to room temperature; freefloating sections were first sorted in PBS, then washed for $5 \times 5 \mathrm{~min}$ in PBS, followed by a 1 -h soak in blocking solution: PBS $+10 \%$ donkey serum (DS; Millipore) and $0.3 \%$ Triton X-100 (Tx; Millipore). Sections were incubated for $18 \mathrm{~h}$ at room temperature in rabbit anti-cFos (F7799, lot:105M4831V; Sigma-Aldrich) diluted 1:2000 in PBS + 5\% DS and $0.3 \% \mathrm{Tx}$ (diluent). Following primary incubation, sections were washed $4 \times 5 \mathrm{~min}$ in PBS $+0.5 \% \mathrm{DS}$, and then incubated for $2 \mathrm{~h}$ at room temperature in Alexa Fluor donkey anti-rabbit 680 (Life Technologies) diluted 6:1000 in diluent. Sections were washed $3 \times 10 \mathrm{~min}$ in PBS, followed by a 30 min incubation in NeuroTrace 500/525 Green Fluorescent Nissl (NT) diluted 1:200 in PBS. Following a final, 10-min PBS wash, sections were mounted onto chrome alum-subbed slides and coverslipped with Prolong Gold Antifade reagent with 4,6-diamidino-2-phenylindole (DAPI; Life Technologies) and stored at $4^{\circ} \mathrm{C}$ until microscopy.

\section{Image acquisition and anatomy}

All images were collected at $10 \times$ with $568-\mathrm{nm}$ resolution using a Leica SP8 scanning confocal microscope. cFos-ir neurons were visualized using a 680-nm laser line; DAPI and NT were visualized using 405- and 490-nm laser lines, respectively. The intensity of each laser line was identical for all images, and tissue was scanned at 12 separate $z$ planes spaced $2.41 \mu \mathrm{m}$ apart. When more than one confocal image was needed to capture the expanse 
of a nucleus (LS, mPOA, VMH, PAG) or adjacent nuclei (i.e., $P V N$ and $A H$ ), images were automatically merged using Leica Application Suite X software (Leica Microsystems). In instances where tissue was damaged, the next available section was used.

SBN regions were identified based on cytoarchitecture at approximate rostral-caudal levels relative to bregma as per the mouse brain atlas (Paxinos and Franklin, 2004). The LS (Fig. 1G) was collected beginning at the bifurcation of the AC (bregma $0.38 \mathrm{~mm}$ ) and continued for three consecutive sections. We captured the medial division of the BNST starting at the level where the bifurcation of AC begins to close (bregma $0.26 \mathrm{~mm}$ ). BNST (Fig. 1H) was sampled bilaterally in two consecutive sections within boundaries established by the lateral ventricle and the stria terminalis (lateral), the fornix (medial), and ventrally by the AC. Sampling for mPOA (Fig. 1/) began at the closure of $A C$ (bregma $0.14 \mathrm{~mm}$ ) and continued for two consecutive sections. PVN was shot at three consecutive sections beginning at the appearance of a triangular cluster of neurons immediately adjacent to the dorsal aspect of the third ventricle (bregma $-0.70 \mathrm{~mm}$ ). We collected $\mathrm{AH}$ beginning at the second level of PVN (bregma -0.82 $\mathrm{mm}$; Fig. 1J) and continued for three consecutive sections. The final level of $\mathrm{AH}$ overlapped with the first section where sampling for $\mathrm{VMH}$ began (bregma -1.22) and continued for two to three sections (Fig. 1K). PAG (Fig. 1L) was collected for three consecutive sections beginning approximately at bregma $-4.24 \mathrm{~mm}$.

Z stacks for laser lines 405 (DAPI), 490 (NT), and 680 (cFos-ir) were projected using the maximal intensity function in Fiji (National Institutes of Health; Schindelin et al., 2012), and saved as .tif files before analysis. A single observer blind to animal identity and treatment group performed all image analyses and microscopy.

\section{Cell counting}

Regions of interest (ROIs) were drawn around the boundaries of SBN nodes based on cytoarchitecture (Fig. 1G$L$ ) and saved using the Fiji ROI manager. cFos-ir neurons were quantified using custom macros in ImageJ as follows. First, background was subtracted from cFos images using the rolling ball function with a radius of 50 pixels. ROIs derived from NT images were transferred to the corresponding cFos channel. Using the internal clipboard function, we created a new image containing only the selected ROI. Tsai's moments threshold was applied using Fiji's Auto Threshold plugin v1.17. The thresholded image was then made binary, the watershed function was applied, and the analyze particles function was run thresholding out objects with fewer than 75 pixels and a circularity $<0.15$. Cell counts were normalized by multiplying the total number of cFos-ir neurons in each region by 100 divided by the sum of the areas of the ROI(s) from which they were obtained.

\section{Statistics}

Inferential statistics were performed in JMP Pro version 14 (SAS Institute Cary) with an $\alpha=0.05$, or GraphPad
Prism version 8 . We used repeated measures multivariate ANOVA (MANOVA) to test the between-subject effects of housing (SOC vs ISO) and drug treatment (SAL vs FEN vs pCPA) on the within-subjects measure of cFos-ir neurons $/ 100 \mu \mathrm{m}^{2}$ across seven nodes of the SBN. We found main effects of housing and drug treatment, as well as a significant housing-by-drug interaction (see Results). We followed MANOVA with a series of linear mixed model analyses with housing and drug treatment as fixed effects to test for group differences in cFos-ir neurons within each SBN node. Our model included IHC run as a random effect to control for potential variation introduced by separate IHC procedures. As SOC mice were housed in groups of 3 , we also included cage as a random effect to control for potential within-cage influence on cFos expression. Post hoc differences between groups were assessed via independent $t$ tests where applicable.

Next, we performed pair-wise correlations on the number of cFos-ir neurons to test for functional relationships between SBN nodes within each of our six treatment groups; $p$ values obtained from Pearson coefficients were corrected for multiple comparisons using the two-stage linear step-up procedure of Benjamini, Krieger, and Yekutieli (Benjamini et al., 2006). To test for differences in the distribution of internodal correlations between groups, we performed principal components analysis (PCA) on the covariation matrix derived from these data.

Network analyses were performed on within-group correlations using Gephi open source network analysis and visualization software version 0.9.2 (Bastian et al., 2009). Functional relationships were visualized as unweighted, undirected graphs using the ForceAtlas2 algorithm, which spatially distributes nodes based on the overall strengths of each node's correlations (Bastian et al., 2009; Jacomy et al., 2014). Graphs were subsequently filtered so that non-significant edges (i.e., correlations $p>0.05$ ) were excluded from visualization. The overall relatedness of any given region is indicated not only by its shared edges, but by its position relative to other nodes. In order to quantitatively describe functional relationships among groups, we performed three separate graph analyses in Gephi. First, we calculated network density, the number of significant intraregional correlations as a proportion of the total number of possible correlations, for each treatment group. This analysis, a graph-based supplement to our strength of correlation analysis (see Results), quantifies the overall functional connectivity of the SBN in each treatment group. Next, for each treatment group we calculated the average clustering coefficient as the likelihood for any pair of a node's functional connected neighbors to be connected to each other (Watts and Strogatz, 1998; Fornito et al., 2016). Finally, we performed community analysis which parses nodes into highly interconnected subgroups which is indicative of functional commonality (Fornito et al., 2016).

\section{Results}

Repeated measures MANOVA found significant effects of housing $\left(F_{(6,36)}=4.45, p=0.002\right)$ and drug treatment $\left(F_{(12,74)}=5.71, p<0.0001\right)$, as well as a significant 
Table 1: Summary of mixed linear models

\begin{tabular}{|c|c|c|c|c|c|c|c|c|c|}
\hline Source & DF & $F$ & $p$ & Variance component & Estimate & SE & $95 \%$ lower & 95\% upper & $p$ \\
\hline \multicolumn{10}{|l|}{ LS } \\
\hline Drug & $2,22.8$ & 15.98 & $<0.0001^{\star \star \star}$ & IHC run & 7.83 & 9.45 & -10.70 & 26.36 & 0.41 \\
\hline \multicolumn{10}{|l|}{ BNST } \\
\hline Housing & $1,14.5$ & 7.23 & $0.02^{*}$ & Cage number & 3.15 & 6.84 & -10.25 & 16.55 & 0.65 \\
\hline Drug & $2,14.5$ & 52.63 & $<0.0001^{\star \star \star}$ & IHC run & 2.80 & 4.31 & -5.64 & 11.24 & 0.52 \\
\hline Housing & $1,20.5$ & 6.67 & $0.02^{*}$ & Cage number & 57.24 & 24.33 & 9.56 & 104.93 & $0.02^{*}$ \\
\hline Drug & $2,20.5$ & 25.34 & $<0.0001^{\star \star \star}$ & IHC run & 34.86 & 40.78 & -45.08 & 114.80 & 0.39 \\
\hline Housing $\times$ drug & $2,20.5$ & 1.96 & 0.17 & Residual & 28.66 & 10.54 & 15.59 & 69.15 & \\
\hline \multicolumn{10}{|l|}{ PVN } \\
\hline Housing & $1,19.1$ & 10.12 & $0.005^{\star \star}$ & Cage number & 21.13 & 12.54 & -3.44 & 45.70 & 0.09 \\
\hline Drug & $2,19.1$ & 40.99 & $<0.0001^{\star * *}$ & IHC run & 13.95 & 17.01 & -19.39 & 47.29 & 0.41 \\
\hline Housing $\times$ drug & $2,21.2$ & 1.91 & 0.17 & Residual & 24.55 & 6.49 & 15.53 & 44.58 & \\
\hline \multicolumn{10}{|l|}{ VMH } \\
\hline Housing & $1,21.5$ & 2.52 & 0.13 & Cage number & 7.18 & 2.50 & 2.28 & 12.08 & $0.004^{\star *}$ \\
\hline Drug & $2,21.5$ & 14.47 & $<0.0001^{\star \star \star}$ & IHC run & 0.49 & 1.01 & -1.48 & 2.46 & 0.63 \\
\hline $\begin{array}{l}\text { Housing } \times \text { drug } \\
\text { PAG }\end{array}$ & $2,21.5$ & 0.06 & 0.94 & Residual & 1.48 & 0.56 & 0.79 & 3.68 & \\
\hline Housing & $1,22.5$ & 6.11 & $0.02^{*}$ & Cage number & 32.71 & 11.90 & 9.39 & 56.04 & $0.01^{* *}$ \\
\hline Drug & $2,22.5$ & 0.91 & 0.42 & IHC run & 5.48 & 8.02 & -10.24 & 21.21 & 0.49 \\
\hline Housing $\times$ drug & $2,22.5$ & 1.45 & 0.26 & Residual & 10.71 & 3.87 & 5.88 & 25.36 & \\
\hline
\end{tabular}

Statistically significant main effects and interactions indicated with asterisks: ${ }^{\star} p<0.05,{ }^{\star *} p<0.01,{ }^{\star \star \star} p<0.001$.

housing-by-drug interaction $\left(F_{(12,74)}=3.17, p=0.001\right)$ on number of cFos-ir neurons $/ 100 \mu \mathrm{m}^{2}$ across seven nodes of the SBN. Next, we performed linear mixed model analyses with housing and drug treatment as fixed factors, controlling for random effects of IHC run and cage. Below we report only $p$ values for linear mixed models; a complete summary can be found in Table 1. Arithmetic means for housing and drug treatments are reported in Table 2. Results from the applicable post hoc comparisons can be found in Extended Data Figure 2-1.

Our model detected effects of housing in the BNST $(p=0.017), \operatorname{mPOA}(p=0.018), \mathrm{PVN}(p=0.005)$, and PAG $(p=0.021)$. ISO mice had higher numbers of cFos-ir neurons in each one of these regions (Tukey's HSD, $p \leq 0.02$ ). We found significant effects of drug treatment in LS $(p<0.0001$; Fig. 2A), BNST $(p<0.0001$; Fig. $2 B)$,
mPOA ( $p<0.0001$; Fig. 2C), PVN ( $p<0.0001$; Fig. $2 D)$, $\mathrm{AH}(p=0.006$; Fig. $2 E)$, and $\mathrm{VMH}(p=0.0001$; Fig. $2 F)$. In each region except for $\mathrm{AH}$, FEN mice had significantly more cFos-ir neurons than both SAL and pCPA mice (Tukey's HSD, $p \leq 0.0007$ ). In AH, FEN mice had significantly more cFos-ir neurons than pCPA mice (Tukey's HSD, $p=0.005$ ), and a trend toward an increase relative to SAL mice (Tukey's HSD, $p=0.054$ ). Interestingly, PAG was the only region where our model did not detect a significant effect of drug treatment ( $p>0.4$; Fig. 2G). Drug effects were dependent on housing conditions as indicated by significant interaction terms in LS $(p=0.018)$ and PVN $(p=0.003)$. In LS, the interaction is driven by an increase in cFos-ir neurons in ISO-pCPA compared with the SOC-pCPA mice. In PVN, the interaction is driven by an almost

Table 2: Summary of group means with housing and drug treatments

\begin{tabular}{|c|c|c|c|c|c|c|c|}
\hline Housing & LS & BNST & $\mathrm{mPOA}$ & PVN & $\mathrm{AH}$ & VMH & PAG \\
\hline Social & $25.25 \pm 3.8$ & $13.6 \pm 5.2$ & $31.58 \pm 6.6$ & $15.97 \pm 4.1$ & $15.24 \pm 2.0$ & $4.48 \pm 2.1$ & $12.50 \pm 0.6$ \\
\hline Isolated & $26.23 \pm 2.5$ & $17.56 \pm 5.3^{*}$ & $40.65 \pm 10.2^{*}$ & $23.39 \pm 10.4^{\star *}$ & $16.00 \pm 2.1$ & $6.17 \pm 2.1$ & $18.42 \pm 2.6^{*}$ \\
\hline \multicolumn{8}{|c|}{ Summary of arithmetic group means of cFos-ir neurons within drug treatments $\pm \mathrm{SE}$} \\
\hline Drug treatment & LS & BNST & $\mathrm{mPOA}$ & PVN & $\mathrm{AH}$ & VMH & PAG \\
\hline SAL & $22.95 \pm 1.2$ & $10.32 \pm 1.0$ & $27.73 \pm 0.1$ & $12.71 \pm 1.1$ & $14.79 \pm 1.5$ & $3.587 \pm 0.5$ & $13.83 \pm 0.9$ \\
\hline FEN & $31.36 \pm 0.9^{\star *}$ & $26.0 \pm 2.1^{\text {*** }}$ & $52.59 \pm 8.1^{\star \star \star *}$ & 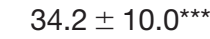 & $19.06 \pm 1.0 *$ & $9.458 \pm 0.9^{\text {***}}$ & $15.2 \pm 2.0$ \\
\hline $\mathrm{pCPA}$ & $22.9 \pm 3.6$ & $10.42 \pm 2.9$ & $28.02 \pm 5.4$ & $12.13 \pm 1.0$ & $13.02 \pm 1.6$ & $2.933 \pm 1.2$ & $17.35 \pm 6.0$ \\
\hline
\end{tabular}

Values expressed as arithmetic mean \pm SEM. Statistically significant differences group means indicated with asterisks: ${ }^{\star} p<0.05,{ }^{* \star} p<0.01,{ }^{\star \star \star} p<0.001 ; p$ values corrected for multiple comparisons (Benjamini et al., 2006). Complete post hoc comparisons can be found in Extended Data Figure 2-1. 
A

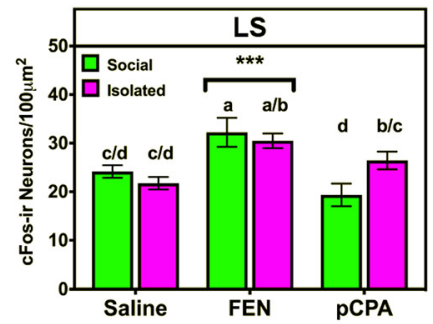

B

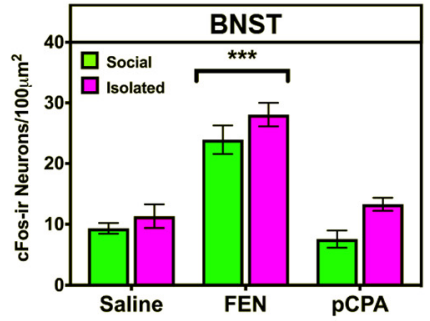

C

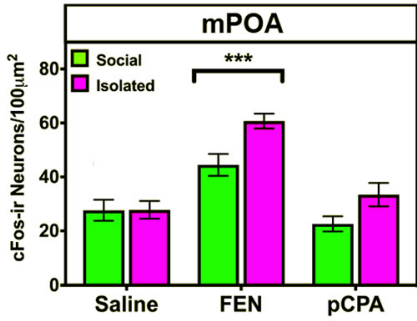

D

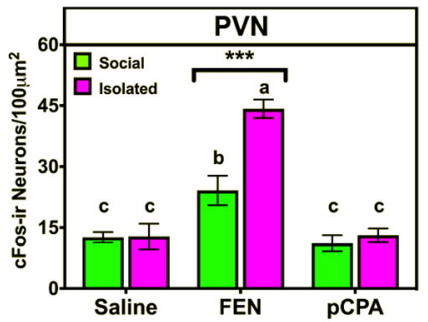

E

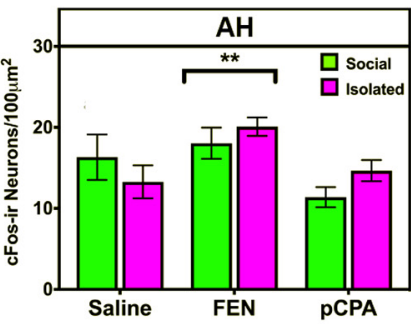

F

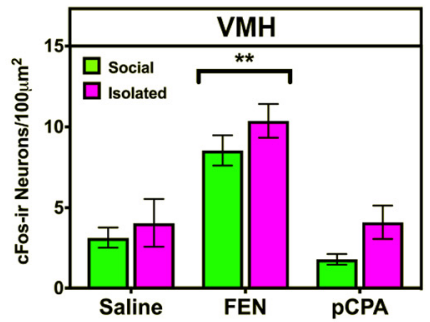

G

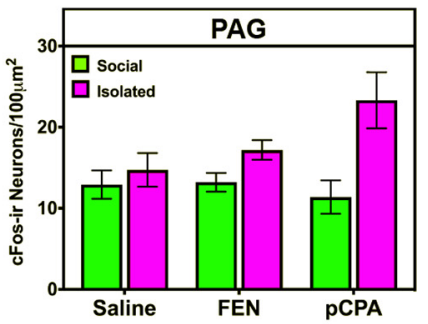

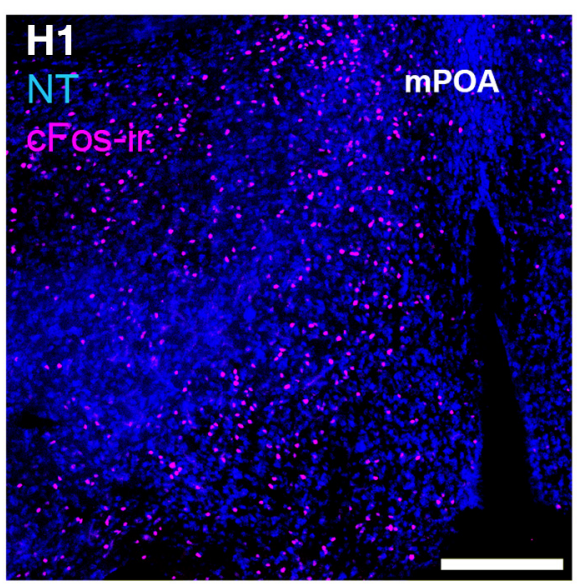
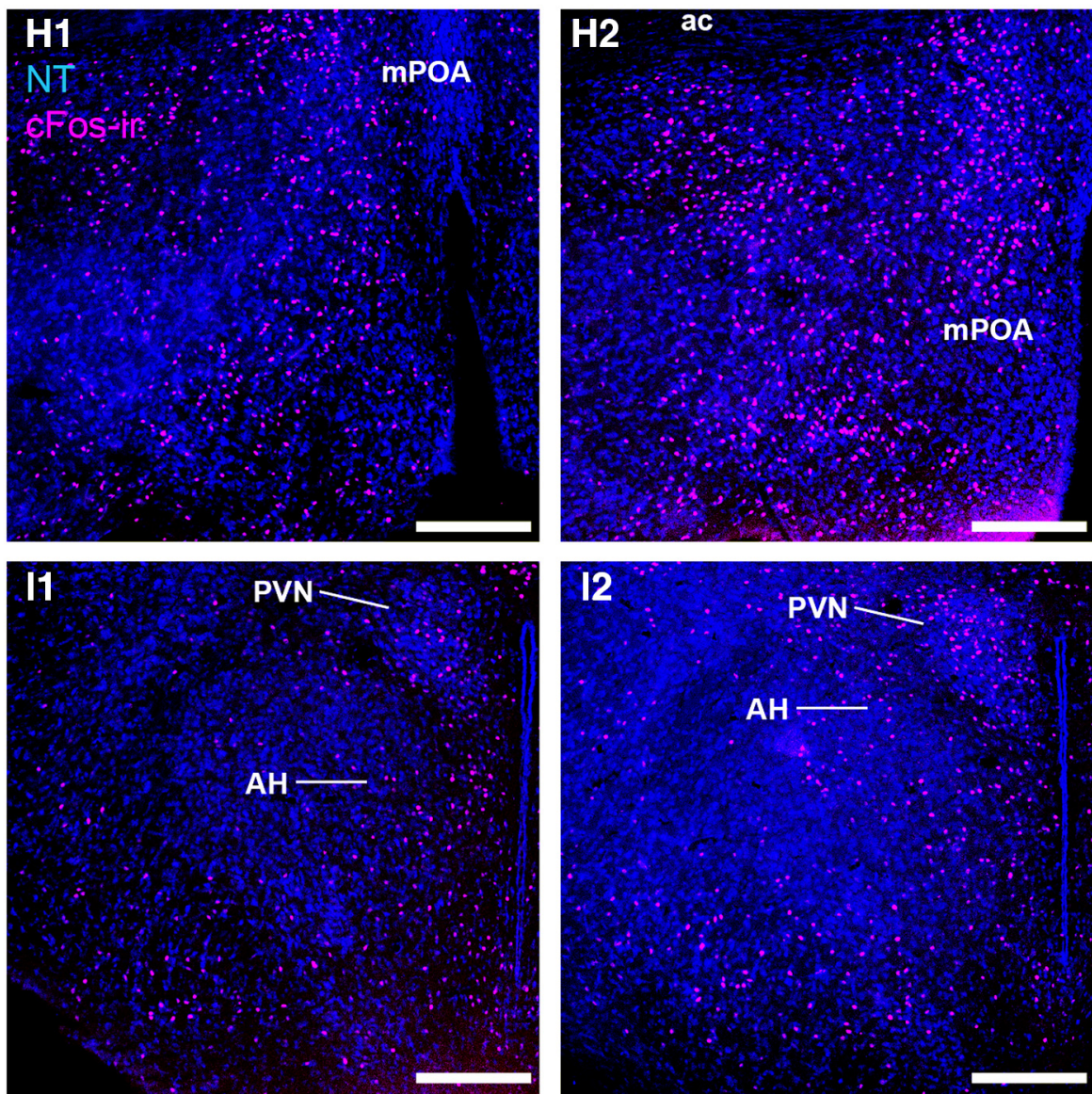

Figure 2. Summary linear mixed model analysis of cFos-ir within nodes of the SBN. A-G, All data are represented as arithmetic mean \pm SEM between socially housed mice (green) and socially isolated mice (magenta). Asterisks represent main effects of drug treatment, ${ }^{* *} p<0.001,{ }^{* * *} p<0.0001$. Letters indicate post hoc differences (independent $t$ test, $p$ values corrected for multiple comparisons) in the case of housing by drug interaction. $\boldsymbol{H}, \boldsymbol{I}$, Representative photomicrographs in mPOA $(\boldsymbol{H})$ and PVN $(\boldsymbol{I})$ showing the effects of FEN on cFos-ir in socially reared $(\boldsymbol{H 1} / \mathbf{I 1})$ or socially isolated $(\boldsymbol{H} 2 / \mathbf{I})$ mice. NT, blue; cFos-ir, magenta. Scale bar: $250 \mu \mathrm{m}$. Please see Extended Data Figure 2-1 for a complete summary of post hoc analyses for linear mixed models performed within each SBN region. 
A

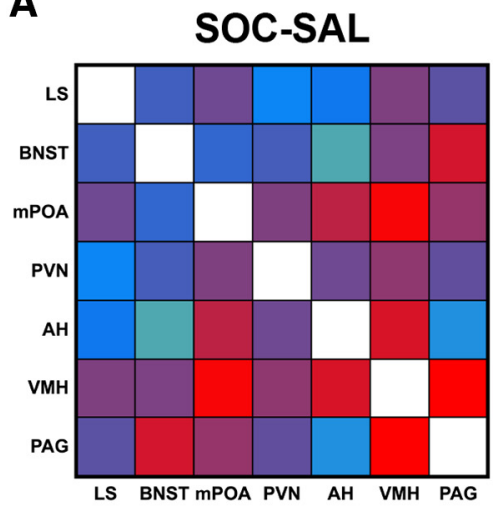

D

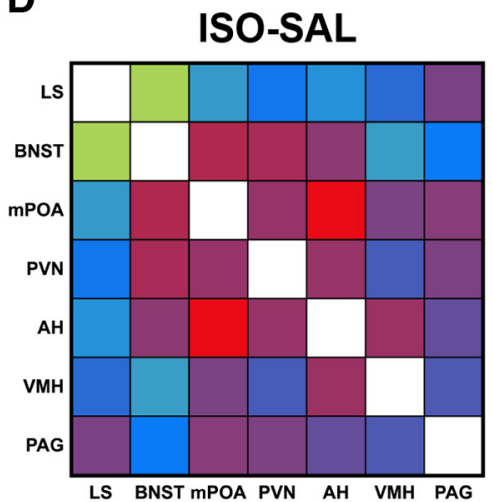

B

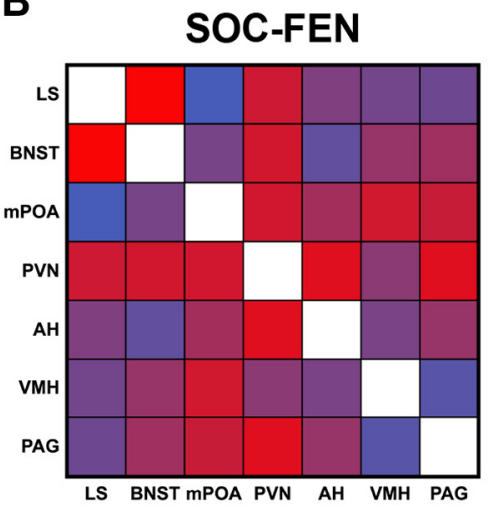

E

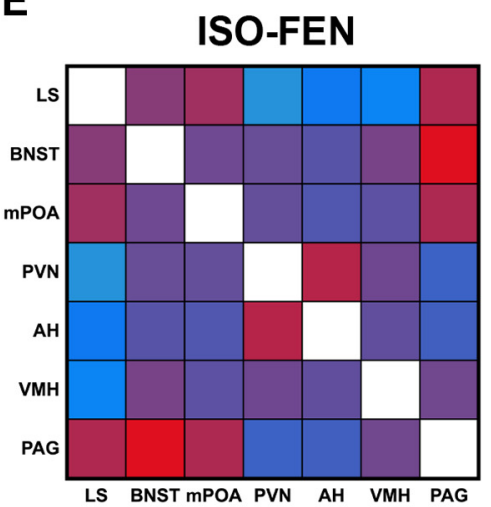

C

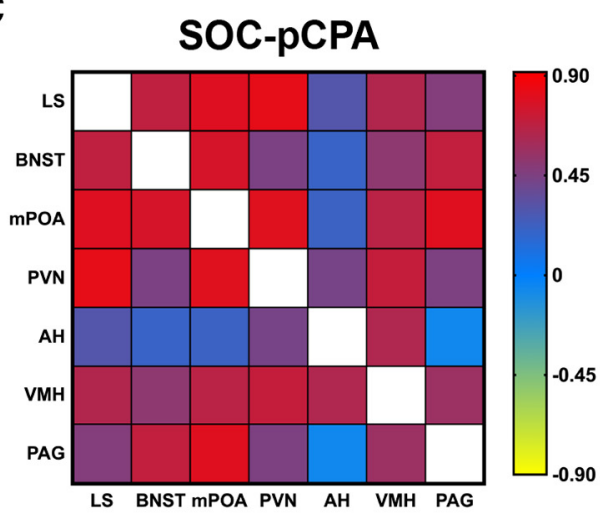

$\mathbf{F}$

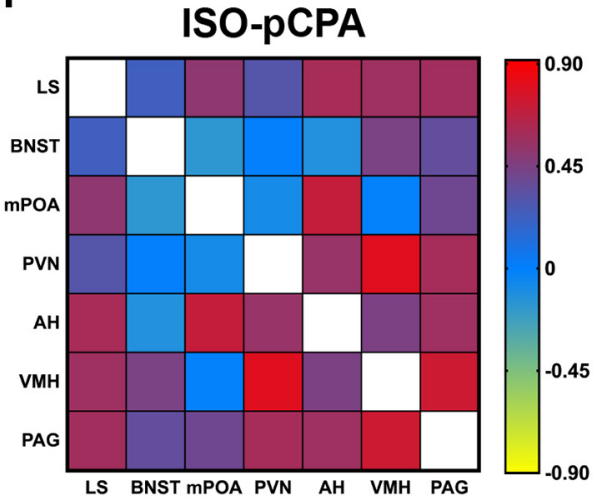

G

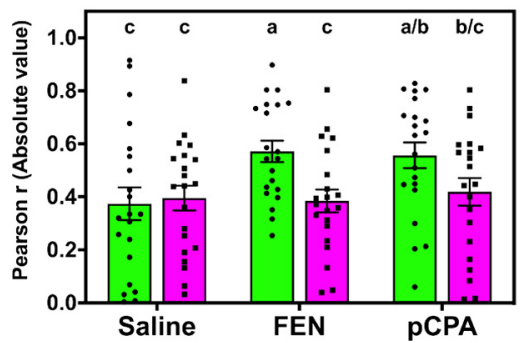

H

\begin{tabular}{|c|c|c|c|c|c|}
\hline PC & Eigenvalue & Percent & Region & PC1 & PC2 \\
\hline 1 & \begin{tabular}{|r|}
543.53 \\
\end{tabular} & $\begin{array}{l}6.77 \\
\end{array}$ & LS & 0.217 & 0.203 \\
\hline 2 & 56.22 & 7.94 & BNST & 0.345 & -0.165 \\
\hline 3 & 39.69 & 5.61 & mPOA & 0.682 & 0.34 \\
\hline 4 & 30.35 & 4.29 & PVN & 0.553 & -0.548 \\
\hline 5 & 20.82 & 2.94 & $\mathrm{AH}$ & 0.173 & 0.026 \\
\hline 6 & 12.27 & 1.73 & VMH & 0.144 & -0.019 \\
\hline 7 & 5.09 & 0.72 & PAG & 0.115 & 0.716 \\
\hline
\end{tabular}

I

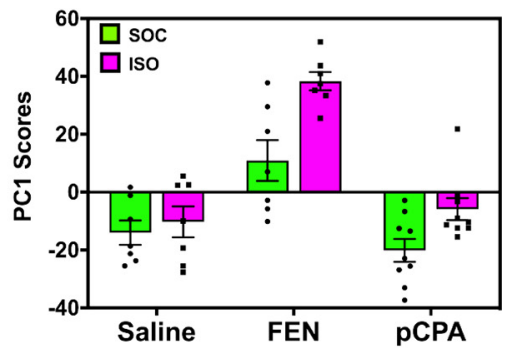

Figure 3. Correlated patterns of cFos-ir are dependent on social experience. $\boldsymbol{A}-\boldsymbol{F}$, Heatmap matrices represent pairwise correlations between cFos-ir neurons with SBN nodes (boxes); colors indicate Pearson correlation coefficients. White boxes are self-correlations $(r=1)$; data are mirrored above and below the diagonal. $\mathbf{G}$, Comparison between the absolute value of Pearson correlation coefficients between groups. Data are represented as mean $\pm \mathrm{SEM}$; letters indicate post hoc differences (independent $t$ test, $p$ values corrected for multiple comparisons). $\boldsymbol{H}$, Results of PCA on covariation matrix derived from $\boldsymbol{A}-\boldsymbol{F}$. $\boldsymbol{I}$, Distribution of PC1 scores between groups; data are represented as mean \pm SEM. Please see Extended Data Figure 3-1 for a complete summary of pairwise correlation statistics.

twofold increase in cFos-ir neurons in ISO-FEN compared with SOC-FEN mice.

We found a main effect of housing $\left(F_{(1,47)}=5.97\right.$, $p=0.02)$ and a significant housing-by-drug interaction $\left(F_{(2,47)}=6.03, p=0.005\right.$; data not shown) on the number of USVs emitted by focal males during playback. The interaction was driven by a significant increase in USV production in ISO-SAL compared with SOC-SAL mice (Tukey's HSD, $p=0.004)$. There were no differences in USV production between SOC and ISO males in either the FEN or pCPA groups $(p>0.8)$. Interestingly, there were no relationship between USV production and cFos-ir within the SBN of any treatment group.

As the SBN comprises a reciprocally connected anatomic network (Hahn et al., 2019), we tested whether correlations of neural activity markers between nodes (i.e., functional connectivity) varied between treatment groups. Figure 3 summarizes these data as heatmap matrices based on the Pearson $r$ values of each pairwise correlation. A detailed summary of pairwise correlations of cFosir neurons between SBN nodes within each of our six treatment groups can be found in Extended Data Figure 
3-1. Cursory analysis of the heatmaps suggested that SOC-FEN (Fig. 3B) and SOC-pCPA (Fig. 3C) had more relatively strong correlations (i.e., more functional connectivity) than all other treatment groups. To test this, we used two-way ANOVAs on the absolute value of Pearson $r$ scores of each treatment group (Tanimizu et al., 2017). We found a main effect of housing $\left(F_{(1,120)}=6.33\right.$, $p=0.013$; Fig. 3G), where SOC mice had larger Pearson $r$ values than ISO mice (Tukey's HSD, $p=0.013$ ). Drug treatment $\left(F_{(2,120)}=2.68, p=0.07\right)$ and housing-by-drug interaction $\left(F_{(2,120)}=2.45, p=0.09\right)$ approached but did not reach statistical significance.

While we did not find a statistically significant effect of drug treatment on the strength of internode correlations, we hypothesized that pharmacologically increasing or systemically depleting available serotonin would differentially affect the distribution of functional relationships. We thus performed PCA on the covariation matrix generated by cFos-ir counts. PC1 had an eigenvector of 543.53, which accounted for over $76 \%$ of the variation and contained eigenvalues most strongly loaded by mPOA (0.682) and PVN (0.553; Fig. $3 H$ ). We analyzed PC1 scores between groups via two-way ANOVA and found main effects of housing $\left(F_{(1,41)}=14.11, p<0.001\right)$ and drug treatment $\left(F_{(2,41)}=\right.$ 39.28, $p<0.0001$; Fig. $3 /)$, but no significant interaction $\left(F_{(2,41)}=2.43, p=0.10\right)$. FEN mice had divergent and significantly different (Tukey's HSD, $p<0.0001$ ) PC1 scores from both SAL and FEN mice; Thus, despite having similar overall Pearson $r$ values, the distribution of correlations among SBN nodes was different between drug treatment groups.

We visualized the distributions of correlations using the ForceAtlas2 algorithm in Gephi, and filtered the resulting graphs to exclude non-significant edges (i.e., correlations $p>0.05$ ) from visualization (Bastian et al., 2009; Jacomy et al., 2014). There were visible differences between groups in functional network structure: ISO mice have fewer significant functional relationships than SOC mice, which is indicated by relatively few connections between nodes (Fig. 4A-F). For example, cFos-ir neurons in the $\mathrm{PVN}$ of ISO-FEN mice is significantly correlated with $\mathrm{AH}$; thus, PVN shares an edge with only $\mathrm{AH}$ (Fig. 4E). Conversely, in SOC-FEN mice the number of cFos-ir neurons in the PVN is significantly correlated with LS, BNST, $\mathrm{mPOA}, \mathrm{AH}$, and PAG; thus, PVN shares edges with each of these regions (Fig. 4B). Further, the strength of functional relationships is indicated by the closeness of nodes in space. In SOC-pCPA mice, the significant correlation between cFos-ir in MPOA and BNST is indicated not only by a shared edge, but their relative adjacency (closeness) in space (Fig. 4C). In ISO-pCPA mice, there was a relatively weak, non-significant correlation between cFos-ir in $\mathrm{mPOA}$ and BNST which is reflected by a relatively large distance between these nodes in space (Fig. 4F).

We quantifiably described the network organization of the above graphs by performing three separate graph analyses. First, we quantified the number of significant functional relationships as a proportion of total possible functional relationships (i.e., functional density; Fornito et al., 2016). In each of the three SOC treatment groups, there was an over twofold increase in functional density compared with their ISO counterparts (Fig. 4G). While increased density indicates that there is more functional connectivity between regions in SOC mice, this metric tells us little about the nature of these correlations (Bullmore and Sporns, 2009). Importantly, do correlated nodes go on to form (1) additional functional relationships, and/or (2) larger functional modules?

Next, we calculated the clustering coefficient, the average number of connected pairs of a node's connected neighbors of each treatment group. SOC mice had higher clustering coefficients than ISO mice in each treatment group (Fig. $4 H$ ). Indeed, regardless of drug treatment, ISO mice had a clustering coefficient of zero; thus, even when ISO mice have significant correlations between nodes, those regions do not in-turn form additional connections with each other. Our final graph analysis assessed modularity/community structure in SOC and ISO mice. In community structure analysis, the smallest number of communities that can be formed is one, indicating a completely connected group; the maximum number of communities is equal to the number of nodes contained in the analysis, and indicates complete functional segregation. We found that ISO mice formed more communities than their socially reared counterparts in each of the drug treatment groups (Fig. 4/). Together, our results support that functional networks are more disconnected in ISO mice.

\section{Discussion}

Individual experience establishes the backdrop on which current events are interpreted. Early-life social isolation can profoundly affect an animal's behavioral phenotype (Mumtaz et al., 2018), and likely affects how social signals (e.g., vocalizations) are represented in the brain; however, the effects of social isolation on the neural response to rodent vocalizations are relatively unexplored. We tested whether social experience interacts with serotonin signaling to affect IEG expression in the male SBN following playback of female BBVs. FEN robustly increased the number of cFos-ir neurons across all nodes of the SBN except PAG. Housing treatment also affected IEG induction: ISO mice had more cFos-ir neurons in several nodes of the SBN than SOC mice. Despite a generalized increase in cFos-ir, ISO mice had lower functional connectivity among regions than SOC mice. Indeed, functional density, clustering, and community structure remained relatively low in ISO mice despite pharmacological changes in available serotonin. Importantly, drug treatment had little effect on graph analyses in ISO mice and facilitated network measures in SOC mice.

\section{Social experience interacts with serotonin signaling to affect neural responses in the SBN}

IEG mapping has established that early-life stressors alter neural activity markers at the level of individual SBN nodes. Chronic social subjugation decreased cFos expression in the LS, PVN, and PAG following openarm exposure in male mice (Singewald et al., 2009). In rats, postweaning social isolation increased both aggression and cFos-ir neurons in the BNST and PVN (but 
A

Social-Saline

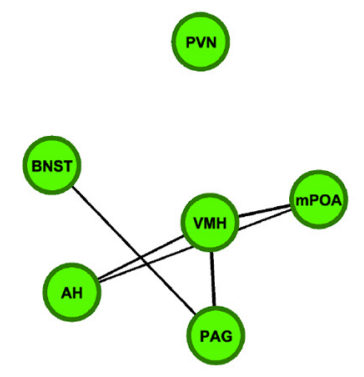

(L)

D

Isolated-Saline

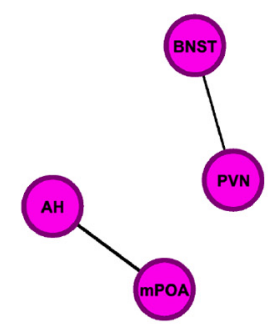

(VMH)

G

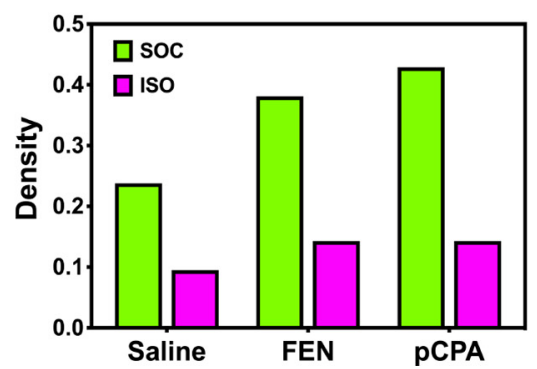

B

Social-Fenfluramine

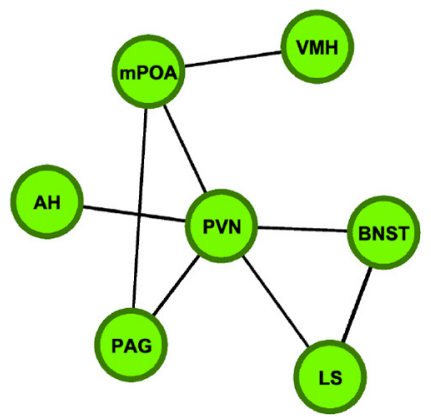

E

Isolated-Fenfluramine
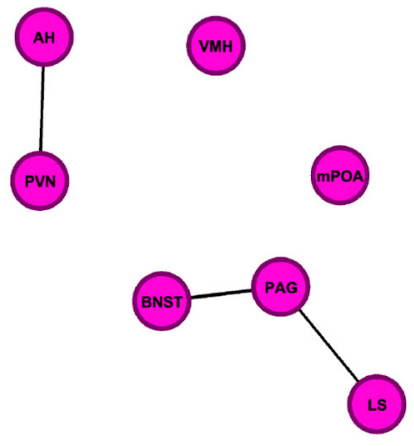

H

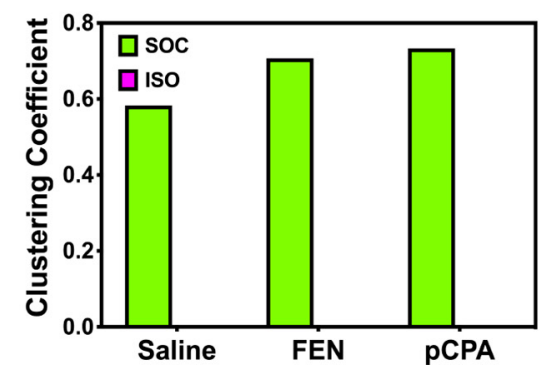

C Social-pCPA

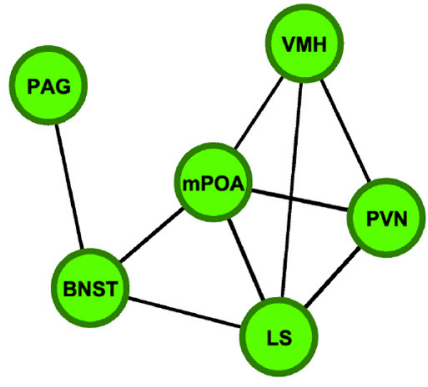

$\mathbf{F}$

Isolated-pCPA

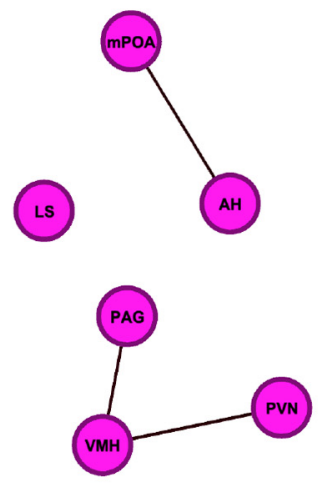

I

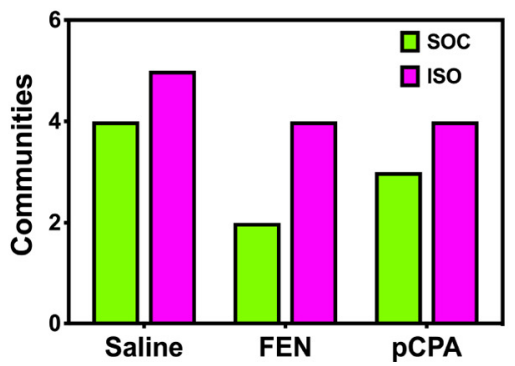

Figure 4. Correlated patterns of activity form different functional networks between treatment groups. A-F, Individual nodes are represented as green (SOC) or magenta (ISO) circles. The spatial distribution of nodes is determined by their individual strengths of correlation (Jacomy et al., 2014). Lines (edges) connecting nodes are indicative of statistically significant Pearson $r$ values $(p<0.05$ ); non-significant edges $(p>0.05)$ are excluded from graphs. G-I, Network measures vary between SOC and ISO mice. G, SOC mice (green) have denser functional networks than ISO mice (magenta). $\boldsymbol{H}$, SOC mice have higher clustering coefficients than ISO mice, whose clustering coefficient is zero in each drug treatment group. $\mathbf{I}$, SOC mice formed fewer thus more densely populated functional communities than ISO mice.

not LS or PAG) following a resident-intruder paradigm (Toth et al., 2012). Despite structural interconnectedness, we found that changes in the cFos response in the SBN of ISO mice was not global: BNST, mPOA, $\mathrm{PVN}, \mathrm{VMH}$, and PAG had increased cFos-ir neurons, whereas LS and AH did not. Interestingly, the direction (i.e., an increase) of the cFos response was similar in each region affected in ISO mice.
The effects of housing on cFos-ir neurons were modulated by drug treatment. Similar to previous studies ( $\mathrm{Li}$ and Rowland, 1993), we found that FEN increased cFos-ir neurons in all nodes of the SBN except for PAG. However, the effects of FEN were not homogenous across housing treatments: cFos-ir neurons were increased to a greater extent in the PVN and mPOA of ISO-FEN compared with SOC-FEN mice (Fig. 3C,D). Conversely, we found no 
difference in cFos-ir in the PVN of pCPA mice regardless of social experience, an observation consistent with previous studies in rats (Harbuz et al., 1993; Conde et al., 1998). Serotonin affects neural activity through a combination of excitatory and inhibitory serotonin receptor (5HTR) subtypes (Barnes and Sharp, 1999), and 5-HTR expression patterns are sensitive to social isolation (Schiller et al., 2003; Bibancos et al., 2007); thus, the net effect of serotonergic manipulations is likely driven by complex excitatory and inhibitory interactions within and between each node of the SBN. Importantly, the increase in cFos-ir neurons was not observed exclusively in ISOFEN mice: ISO-pCPA mice had more cFos-ir neurons in LS, BNST, mPOA, and PAG than SOC-pCPA mice.

Together, we found heterogenous effects of both pharmacology and housing conditions on the number of cFosir neurons within nodes of the SBN. An a priori assumption is that the SBN is a structurally interconnected network within which the patterns of functional connectivity are indicative of behavioral context (Newman, 1999; Goodson, 2005; Goodson and Kabelik, 2009). We therefore tested whether functional network measures differed within the SBN of SOC or ISO mice.

\section{Social experience affects functional connectivity in the SBN}

The term functional connectivity, defined as "statistical dependencies among remote neurophysiological events" (Friston, 2011), has been used extensively in human fMRI studies to describe activity patterns in resting and pathologic states. This statistical phenomenon appears to be a crucial component to adaptive social processes across multiple species, including humans (Lynall et al., 2010). IEG mapping in non-traditional vertebrate model systems demonstrates functional connectivity between neuromodulatory systems/circuits (including the SBN) during vocal-acoustic processing in fishes (Petersen et al., 2013; Ghahramani et al., 2018) and frogs (Hoke et al., 2005), as well as prosocial and aggressive behavior in fishes (Weitekamp and Hofmann, 2017; Butler et al., 2018) and lizards (Kabelik et al., 2018). In male prairie voles (Microtus ochrogaster), oxytocin receptor antagonists reduce functional connectivity within the SBN and attenuate partner preference behavior (Johnson et al., 2016). In mice, Tanimizu et al. (2017) demonstrated that functional connectivity between memory-associated regions (including nodes of the SBN) was increased following a social learning task. Finally, different clusters of functional relationships in the SBN are observed in subordinate mice who maintain their beta status compared with those who ascend through the social hierarchy (Williamson et al., 2019).

We found that functional connectivity was decreased in ISO relative to SOC mice following playback of female BBVs. Importantly, these results were not because of a global increase in IEG induction as ISO mice tended to have more cFos-ir neurons than SOC mice. That functional connectivity is disrupted in the SBN of ISO mice represents an important foundation from which to develop models of how variation in functional network architecture relates to variation in aberrant behavioral (Keesom et al., 2017b; Manouze et al., 2019) and neural phenotypes (Keesom et al., 2017a, 2018) following early-life social stress.

We further analyzed patterns of functional connectivity by PCA and found that FEN mice had positive average PC1 scores, whereas SAL and PCPA mice had negative values (Fig. 4/). Thus, variation in the distribution of functional relationships differs following acutely increasing (i.e., with FEN) or systemically depleting (i.e., with pCPA) serotonin. Interestingly, the general direction of these relationships was consistent within drug treatment regardless of housing conditions. Therefore, while overall functional connectivity may be preferentially modulated by social experience, serotonin signaling drives variation in the nodes that are functionally coupled. However, the extent to which the effects of social experience and serotonin signaling are independent of each other remains unknown.

\section{Individual nodes disproportionately affect functional connectivity}

PCA revealed that individual nodes disproportionately affect variation in functional connectivity. We found that eigenvectors within PC1 were most heavily loaded by mPOA and PVN. As mPOA and PVN underlie different functions, they may also drive variation in circuit-level metrics in different manners. MPOA has increased IEG induction following playback of social vocalizations in frogs and songbirds (Hoke et al., 2005; Maney et al., 2008). In mice, $\mathrm{mPOA}$ is a crucial site for affective-olfactory integration (Dhungel et al., 2011; McHenry et al., 2017), and activity in $\mathrm{mPOA}$ coincides with sociosexual investigation and facilitates mounting behavior (Wei et al., 2018). As the behavioral response to vocal signals (i.e., BBVs) is modulated by olfactory stimuli (Grimsley et al., 2013; Seagraves et al., 2016; Ronald et al., 2020), mPOA is in a functional anatomic position to integrate multisensory stimuli and effect circuit-level responses to vocal signals (Kohl et al., 2018). However, to our knowledge no studies have directly investigated $\mathrm{mPOA}$ involvement in rodent vocal processing.

We found that the number of cFos-ir neurons in the PVN is not only increased in ISO mice, but contributes a significant amount of variation to functional relationships within the SBN. Within the PVN, dysregulation of corticotropin-releasing factor (CRF) neurons which modulate the hypothalamic-pituitary-adrenal axis contributes to cardiovascular disease and impaired immune function in animal models of chronic social isolation as well as in persons with early-life social trauma (Heim and Nemeroff, 2001; McEwen, 2003; Cacioppo et al., 2015). Further, chemically heterogeneous PVN neurons underly different suites of behaviors: activating CRF neurons in PVN drives conditioned place aversion (Kim et al., 2019), whereas activating oxytocin neurons drives pup retrieval behavior in response to USVs (Marlin et al., 2015). Elucidating the chemical phenotypes and projection profiles of isolationsensitive neurons in the PVN (and SBN in general) will be crucial to understanding the mechanisms through which different nodes affect functional relationships within the SBN. 


\section{Network measures are lower in ISO relative to SOC mice}

Variation in functional relationships includes reduced functional connectivity in ISO mice. Further, we found decreased network density and clustering in ISO mice, which is consistent with observations that similar reductions are observed in persons with schizophrenia (Lynall et al., 2010) and depression (Wise et al., 2017). Similarly, functional dysconnectivity within the SBN may provide a partial mechanism for decreased social competence observed in mice raised in social isolation (Keesom et al., 2017b). Investigating the mechanisms that drive variation in functional dysconnectivity will contribute to a better understanding of the diverse array of physiological and behavioral consequences observed in animals following social isolation and other early-life stressors (Heim and Nemeroff, 2001; Mumtaz et al., 2018). As neither FEN nor pCPA were sufficient to facilitate an increase in network measures in ISO mice, it is critically important to consider variation in an individual's contextual state (i.e., social history, physiological condition, pathologic severity, etc.) when designing experiments and interpreting the results of psychoactive reagents on animal or human subjects (Bartz et al., 2011).

\section{Technical considerations}

This study does not directly compare the cFos response in the SBN following playback of BBVs to a nonsocial acoustic stimulus (i.e., pure tones) or a silent condition. Playback of social vocalizations is sufficient to increase the cFos expression relative to tones in in the PAG of rats (Ouda et al., 2016), and relative to ambient environmental noise in multiple SBN nodes in the midshipman fish (Porichthys notatus; Petersen et al., 2013; Ghahramani et al., 2018). However, a lack of playback in the current study would not necessarily be a behaviorally neutral condition, given that silence could have a very different significance for socially housed versus isolated males. Likewise, mouse vocal signals encompass a wide range of frequencies and nonlinear structures (Holy and Guo, 2005; Grimsley et al., 2013; Lupanova and Egorova, 2015; Finton et al., 2017; Niemczura et al., 2020) and some commonly used 'control' sounds can evoke behavioral responses similar to call playback (K. Hood and L. Hurley, unpublished observations). In comparison to silence or other non-vocal sounds, playback of BBVs is a condition with a behavioral salience that is relatively well-understood. BBVs correspond to fewer male mounts of females in observational studies (Finton et al., 2017). Playback of BBVs alters the number of USVs as well as the numbers of males making USVs (Niemczura et al., 2020; Ronald et al., 2020). BBVs are therefore suppressive to some types of social behaviors, creating a defined behavioral context in the current study. However, we are unable to determine whether the observed cFos response in the SBN of SOC and ISO mice is selective for social vocalizations (Maney et al., 2006, 2008), whether functional connectivity is an emergent response to socially salient stimuli (Hoke et al., 2005; Ghahramani et al., 2018), or as a potential confound from a novel testing environment (VanElzakker et al., 2008) or as a direct result of social isolation in SOC animals
(Matthews et al., 2016). Further studies are needed to directly test how the SBN responds to social vocalizations, and to probe the consequences of variation in functional connectivity in the production of murine social behaviors.

IEG mapping is limited in that there is no direct relationship with the production of action potentials and the presence of IEG products (including cFos; Clayton, 2000; Kovacs, 2008). Despite its wide use as a proxy for neural activation, the cFos protein is a transcription factor; one interpretation of our data is that instead of an increase in neural activity per se, we are observing an increased potential for neuroplasticity in ISO mice. Further, using cFos-ir as a putative marker for neural activity limits our temporal resolution to the entire 60 -min trial. It is therefore impossible to make causal or directional statements pertaining to functional connectivity. We make no assumption that functional relationships imply structural connectivity, or vice versa. For example, a subset of mPOA neurons monosynaptically project to PVN (Kohl et al., 2018); however, the particular neurons that connect $\mathrm{mPOA}$ and $\mathrm{PVN}$ might not be similarly engaged in our paradigm.

Importantly, because the presence of cFos-ir is an indicator of past neural activity, it may be robust to action potentials that otherwise habituate over the course of a repeated stimulus i.e., auditory playback. For example, whereas neurons in the IC decrease spike rate following repeated presentation of auditory stimuli (Pérez-González et al., 2005), cFos-ir neurons are detectable in the IC following 90 min playbacks of pure-tone stimuli (D'Alessandro and Harrison, 2014). Further, repeated presentation of ultrasonic distress calls increases cFos-ir within auditory and limbic structures in the rat (Ouda et al., 2016). We find it unlikely that the potential for SBN neurons to adapt to repeated playback of BBVs influenced our results. cFos takes $\sim 30 \mathrm{~min}$ to reach detectable changes in expression, and the half-life of the cFos protein is $\sim 45 \mathrm{~min}$ (Kovacs, 2008). The mice used in this study were at least $180 \mathrm{~min}$ removed from being transferred to the lab and $135 \mathrm{~min}$ removed from injections; we would expect cFos induction brought on by these aspects of our experimental design to be negligible.

Systemic pharmacological manipulations likely drive "off-target" effects such that activity markers are influenced not only by serotonin signaling within the SBN, but via direct or polysynaptic modulation of inputs into the SBN. Extrinsic to the SBN, depleting systemic serotonin reduces synaptic densities in the cortex of rats (Chen et al., 1994) and changes glutamate receptor distribution in the amygdala (Tran et al., 2013). Interestingly, functional connectivity/density, clustering, and community structure remain relatively high in SOC-FEN and SOC-pCPA mice despite potential off-target pharmacological and physiological effects (Otchy et al., 2015) resulting from chronically depleting or acutely increasing available serotonin. Manipulating site-specific serotonergic inputs into the SBN will be crucial to untangling the effects of individual nodes on network-level functionality (Ren et al., 2018). Importantly, network measures were also increased in SOC-SAL relative to ISO-SAL mice, suggesting the importance of social experience in the ability to form functional relationships in the SBN. 
In conclusion, FEN and social isolation each broadly increased cFos-ir within individual nodes of the SBN following payback of social vocalizations. Importantly, by extending our analyses from individual nodes to the network level, we found that functional connectivity, clustering, and community structure within the SBN was highly dependent on social experience, whereas patterns of functional connectivity (i.e., which nodes formed functional relationships) were driven more by pharmacological manipulations. Our findings suggest the hypothesis that functional dysconnectivity may underlie psychopathological phenotypes that arise from social isolation and reinforces the importance to move beyond functional analyses limited to individual nodes. We highlight the importance of how laboratory housing conditions (SOC vs ISO) can affect functional neuroanatomical processes in rodents.

\section{References}

Bailey NW, Moore AJ (2018) Evolutionary consequences of social isolation. Trends Ecol Evol 33:595-607.

Barnes NM, Sharp T (1999) A review of central 5-HT receptors and their function. Neuropharmacology 38:1083-1152.

Bartz JA, Zaki J, Bolger N, Ochsner KN (2011) Social effects of oxytocin in humans: context and person matter. Trends Cogn Sci 15:301-309.

Bastian M, Heymann S, Jacomy M (2009) Gephi: an open source software for exploring and manipulating networks. Proceedings of the International AAAI Conference on Web and Social Media.

Beier KT, Gao XJ, Xie S, DeLoach KE, Malenka RC, Luo L (2019) Topological organization of ventral tegmental area connectivity revealed by viral-genetic dissection of input-output relations. Cell Rep 26:159-167.e6.

Benjamini Y, Krieger AM, Yekutieli D (2006) Adaptive linear step-up procedures that control the false discovery rate. Biometrika 93:491-507.

Bibancos T, Jardim D, Aneas I, Chiavegatto S (2007) Social isolation and expression of serotonergic neurotransmission-related genes in several brain areas of male mice. Genes Brain Behav 6:529539.

Bullmore E, Sporns O (2009) Complex brain networks: graph theoretical analysis of structural and functional systems. Nat Rev Neurosci 10:186-198.

Bullmore E, Frangou S, Murray R (1997) The dysplastic net hypothesis: an integration of developmental and dysconnectivity theories of schizophrenia. Schizophr Res 28:143-156.

Butler JM, Whitlow SM, Roberts DA, Maruska KP (2018) Neural and behavioural correlates of repeated social defeat. Sci Rep 8:6818.

Cacioppo JT, Cacioppo S, Capitanio JP, Cole SW (2015) The neuroendocrinology of social isolation. Annu Rev Psychol 66:733-767.

Chen L, Hamaguchi K, Ogawa M, Hamada S, Okado N (1994) PCPA reduces both monoaminergic afferents and nonmonoaminergic synapses in the cerebral cortex. Neurosci Res 19:111-115.

Clayton DF (2000) The genomic action potential. Neurobiol Learn Mem 74:185-216.

Conde G, Renshaw D, Lightman S, Harbuz M (1998) Serotonin depletion does not alter lipopolysaccharide-induced activation of the rat paraventricular nucleus. J Endocrinol 156:245-252.

D'Alessandro LM, Harrison RV (2014) Excitatory and inhibitory tonotopic bands in chinchilla inferior colliculus revealed by c-fos immuno-labeling. Hear Res 316:122-128.

Dailly E, Chenu F, Petit-Demoulière B, Bourin M (2006) Specificity and efficacy of noradrenaline, serotonin depletion in discrete brain areas of Swiss mice by neurotoxins. J Neurosci Methods 150:111115.

Davis R, Faulds D (1996) Dexfenfluramine. Drugs 52:696-724.
Decot HK, Namboodiri VMK, Gao W, McHenry JA, Jennings JH, Lee SH, Kantak PA, Jill Kao YC, Das M, Witten IB, Deisseroth K, Shih Y-YI, Stuber GD (2017) Coordination of brain-wide activity dynamics by dopaminergic neurons. Neuropsychopharmacology 42:615627.

Dhungel S, Urakawa S, Kondo Y, Sakuma Y (2011) Olfactory preference in the male rat depends on multiple chemosensory inputs converging on the preoptic area. Horm Behav 59:193-199.

Dölen G, Darvishzadeh A, Huang KW, Malenka RC (2013) Social reward requires coordinated activity of nucleus accumbens oxytocin and serotonin. Nature 501:179-184.

Finton CJ, Keesom SM, Hood KE, Hurley LM (2017) What's in a squeak? Female vocal signals predict the sexual behaviour of male house mice during courtship. Anim Behav 126:163-175.

Fornito A, Zalesky A, Bullmore E (2016) Fundamentals of brain network analysis. San Diego: Academic Press.

Friston K (2011) Functional and effective connectivity: a review. Brain Connect 1:13-36.

Ghahramani ZN, Timothy M, Varughese J, Sisneros JA, Forlano PM (2018) Dopaminergic neurons are preferentially responsive to advertisement calls and co-active with social behavior network nuclei in sneaker male midshipman fish. Brain Res 1701:177-188.

Giorgi A, Migliarini S, Galbusera A, Maddaloni G, Mereu M, Margiani G, Gritti M, Landi S, Trovato F, Bertozzi SM, Armirotti A, Ratto GM, De Luca MA, Tonini R, Gozzi A, Pasqualetti M (2017) Brain-wide mapping of endogenous serotonergic transmission via chemogenetic fMRI. Cell Rep 21:910-918.

Goodson JL (2005) The vertebrate social behavior network: evolutionary themes and variations. Horm Behav 48:11-22.

Goodson JL (2013) Deconstructing sociality, social evolution and relevant nonapeptide functions. Psychoneuroendocrinology 38:465478.

Goodson JL, Wang Y (2006) Valence-sensitive neurons exhibit divergent functional profiles in gregarious and asocial species. Proc Natl Acad Sci USA 103:17013-17017.

Goodson JL, Kabelik D (2009) Dynamic limbic networks and social diversity in vertebrates: from neural context to neuromodulatory patterning. Front Neuroendocrinol 30:429-441.

Goodson JL, Kingsbury MA (2013) What's in a name? Considerations of homologies and nomenclature for vertebrate social behavior networks. Horm Behav 64:103-112.

Grandjean J, Corcoba A, Kahn MC, Upton AL, Deneris ES, Seifritz E, Helmchen F, Mansuy IM, Mann EO, Rudin M, Saab BJ (2019) A brain-wide functional map of the serotonergic responses to acute stress and fluoxetine. Nat Commun 10:350.

Grimsley JM, Hazlett EG, Wenstrup JJ (2013) Coding the meaning of sounds: contextual modulation of auditory responses in the basolateral amygdala. J Neurosci 33:17538-17548.

Hahn JD, Sporns O, Watts AG, Swanson LWJPotNAoS (2019) Macroscale intrinsic network architecture of the hypothalamus. Proc Natl Acad Sci USA 116:8018-8027.

Hanson JL, Hurley LM (2012) Female presence and estrous state influence mouse ultrasonic courtship vocalizations. PLoS One 7: e40782.

Hanson J, Hurley L (2016) Serotonin, estrus, and social context influence c-Fos immunoreactivity in the inferior colliculus. Behav Neurosci 130:600-613.

Harbuz MS, Chalmers J, De Souza L, Lightman SL (1993) Stress-induced activation of CRF and c-fos mRNAs in the paraventricular nucleus are not affected by serotonin depletion. Brain Res 609:167-173.

Harlow HF, Dodsworth RO, Harlow MK (1965) Total social isolation in monkeys. Proc Natl Acad Sci USA 54:90-97.

Heim C, Nemeroff CB (2001) The role of childhood trauma in the neurobiology of mood and anxiety disorders: preclinical and clinical studies. Biol Psychiatry 49:1023-1039.

Hoke KL, Ryan MJ, Wilczynski W (2005) Social cues shift functional connectivity in the hypothalamus. Proc Natl Acad Sci USA 102:10712-10717. 
Holy TE, Guo Z (2005) Ultrasonic songs of male mice. PLoS Biol 3: e386.

Jacomy M, Venturini T, Heymann S, Bastian M (2014) ForceAtlas2, a continuous graph layout algorithm for handy network visualization designed for the Gephi software. PLoS One 9:e98679.

Johnson ZV, Walum H, Jamal YA, Xiao Y, Keebaugh AC, Inoue K, Young LJ (2016) Central oxytocin receptors mediate mating-induced partner preferences and enhance correlated activation across forebrain nuclei in male prairie voles. Horm Behav 79:8-17.

Kabelik D, Weitekamp CA, Choudhury SC, Hartline JT, Smith AN, Hofmann HA (2018) Neural activity in the social decision-making network of the brown anole during reproductive and agonistic encounters. Horm Behav 106:178-188.

Keesom SM, Sloss BG, Erbowor-Becksen Z, Hurley LM (2017a) Social experience alters socially induced serotonergic fluctuations in the inferior colliculus. J Neurophysiol 118:3230-3241.

Keesom SM, Finton CJ, Sell GL, Hurley LM (2017b) Early-life social isolation influences mouse ultrasonic vocalizations during malemale social encounters. PLoS One 12:e0169705.

Keesom SM, Morningstar MD, Sandlain R, Wise BM, Hurley LM (2018) Social isolation reduces serotonergic fiber density in the inferior colliculus of female, but not male, mice. Brain Res 1694:94103.

Kim J, Lee S, Fang Y-Y, Shin A, Park S, Hashikawa K, Bhat S, Kim D, Sohn J-W, Lin D, Suh GSB (2019) Rapid, biphasic CRF neuronal responses encode positive and negative valence. Nat Neurosci 22:576-585.

Koe BK, Weissman AJ, Therapeutics E (1966) p-Chlorophenylalanine: a specific depletor of brain serotonin. J Pharmacol Exp Ther 154:499516.

Kohl J, Babayan BM, Rubinstein ND, Autry AE, Marin-Rodriguez B, Kapoor V, Miyamishi K, Zweifel LS, Luo L, Uchida N, Dulac C (2018) Functional circuit architecture underlying parental behaviour. Nature 556:326-331.

Kovacs KJ (2008) Measurement of immediate-early gene activationc-fos and beyond. J Neuroendocrinol 20:665-672.

Lee H, Kim DW, Remedios R, Anthony TE, Chang A, Madisen L, Zeng $\mathrm{H}$, Anderson DJ (2014) Scalable control of mounting and attack by Esr1+ neurons in the ventromedial hypothalamus. Nature 509:627-632.

Li BH, Rowland NE (1993) Dexfenfluramine induces Fos-like immunoreactivity in discrete brain regions in rats. Brain Res Bull 31:4348.

Lin D, Boyle MP, Dollar P, Lee H, Lein E, Perona P, Anderson DJ (2011) Functional identification of an aggression locus in the mouse hypothalamus. Nature 470:221-226.

Lupanova AS, Egorova MA (2015) Vocalization of sex partners in the house mouse (Mus musculus). J Evol Biochem Phys 51:324-331.

Lynall M-E, Bassett DS, Kerwin R, McKenna PJ, Kitzbichler M, Muller U, Bullmore E (2010) Functional connectivity and brain networks in schizophrenia. J Neurosci 30:9477-9487.

Maney DL, Cho E, Goode CT (2006) Estrogen-dependent selectivity of genomic responses to birdsong. Eur J Neurosci 23:1523-1529.

Maney DL, Goode CT, Lange HS, Sanford SE, Solomon BL (2008) Estradiol modulates neural responses to song in a seasonal songbird. J Comp Neurol 511:173-186.

Manouze H, Ghestem A, Poillerat V, Bennis M, Ba-M'hamed S, Benoliel JJ, Becker C, Bernard C (2019) Effects of single cage housing on stress, cognitive, and seizure parameters in the rat and mouse pilocarpine models of epilepsy. eNeuro 6:ENEURO.017918.2019.

Marlin BJ, Mitre M, D'amour JA, Chao MV, Froemke RC (2015) Oxytocin enables maternal behaviour by balancing cortical inhibition. Nature 520:499-504.

Matthews GA, Tye KM (2019) Neural mechanisms of social homeostasis. Ann NY Acad Sci 1457:5-25.

Matthews GA, Nieh EH, Vander Weele CM, Halbert SA, Pradhan RV, Yosafat AS, Glober GF, Izadmehr EM, Thomas RE, Lacy GD, Wildes CP, Ungless MA, Tye KM (2016) Dorsal raphe dopamine neurons represent the experience of social isolation. Cell 164:617631.

McEwen BS (2003) Early life influences on life-long patterns of behavior and health. Ment Retard Dev Disabil Res Rev 9:149-154.

McHenry JA, Otis JM, Rossi MA, Robinson JE, Kosyk O, Miller NW, McElligott ZA, Budygin EA, Rubinow DR, Stuber GD (2017) Hormonal gain control of a medial preoptic area social reward circuit. Nat Neurosci 20:449-458.

Mumtaz F, Khan MI, Zubair M, Dehpour AR (2018) Neurobiology and consequences of social isolation stress in animal model-A comprehensive review. Biomed Pharmacother 105:1205-1222.

Muzerelle A, Scotto-Lomassese S, Bernard JF, Soiza-Reilly M, Gaspar P (2016) Conditional anterograde tracing reveals distinct targeting of individual serotonin cell groups (B5-B9) to the forebrain and brainstem. Brain Struct Funct 221:535-561.

Newman SW (1999) The medial extended amygdala in male reproductive behavior. A node in the mammalian social behavior network. Ann NY Acad Sci 877:242-257.

Newman ME, Girvan M (2004) Finding and evaluating community structure in networks. Phys Rev E Stat Nonlin Soft Matter Phys 69: e026113.

Niederkofler V, Asher TE, Okaty BW, Rood BD, Narayan A, Hwa LS, Beck SG, Miczek KA, Dymecki SM (2016) Identification of serotonergic neuronal modules that affect aggressive behavior. Cell Rep 17:1934-1949.

Niemczura AC, Grimsley JM, Kim C, Alkhawaga A, Poth A, Carvalho A, Wenstrup JJ (2020) Physiological and behavioral responses to vocalization playback in mice. Front Behav Neurosci 14:155.

O'Connell LA, Hofmann HA (2012) Evolution of a vertebrate social decision-making network. Science 336:1154-1157.

Otchy TM, Wolff SB, Rhee JY, Pehlevan C, Kawai R, Kempf A, Gobes SM, Ölveczky BP (2015) Acute off-target effects of neural circuit manipulations. Nature 528:358-363.

Ouda L, Jílek M, Syka J (2016) Expression of c-Fos in rat auditory and limbic systems following 22-kHz calls. Behav Brain Res 308:196-204.

Paxinos G, Franklin KB (2004) The mouse brain in stereotaxic coordinates. Houston: Gulf Professional Publishing.

Pérez-González D, Malmierca MS, Covey E (2005) Novelty detector neurons in the mammalian auditory midbrain. Eur $\mathrm{J}$ Neurosci 22:2879-2885

Petersen CL, Hurley LM (2017) Putting it in context: linking auditory processing with social behavior circuits in the vertebrate brain. Integr Comp Biol 57:865-877.

Petersen CL, Timothy M, Kim DS, Bhandiwad AA, Mohr RA, Sisneros JA, Forlano PM (2013) Exposure to advertisement calls of reproductive competitors activates vocal-acoustic and catecholaminergic neurons in the plainfin midshipman fish, Porichthys notatus. PLoS One 8:e70474.

Portfors CV, Perkel DJ (2014) The role of ultrasonic vocalizations in mouse communication. Curr Opin Neurobiol 28:115-120.

Ren J, Friedmann D, Xiong J, Liu CD, Ferguson BR, Weerakkody T, DeLoach KE, Ran C, Pun A, Sun Y, Weissbourd B, Neve RL, Huguenard J, Horowitz MA, Luo L (2018) Anatomically defined and functionally distinct dorsal raphe serotonin sub-systems. Cell 175:472-487.e20.

Rogers-Carter MM, Christianson JP (2019) An insular view of the social decision-making network. Neurosci Biobehav Rev 103:119132.

Ronald KL, Zhang X, Morrison MV, Miller R, Hurley LM (2020) Male mice adjust courtship behavior in response to female multimodal signals. PLoS One 15:e0229302.

Rothman RB, Baumann MH (2002) Serotonin releasing agents: neurochemical, therapeutic and adverse effects. Pharmacol Biochem Behav 71:825-836.

Sangiamo DT, Warren MR, Neunuebel JP (2020) Ultrasonic signals associated with different types of social behavior of mice. Nat Neurosci 23:411-422. 
Schiller L, Jähkel M, Kretzschmar M, Brust P, Oehler J (2003) Autoradiographic analyses of 5-HT1A and 5-HT2A receptors after social isolation in mice. Brain Res 980:169-178.

Schindelin J, Arganda-Carreras I, Frise E, Kaynig V, Longair M, Pietzsch T, Preibisch S, Rueden C, Saalfeld S, Schmid B, Tinevez JY, White DJ, Hartenstein V, Eliceiri K, Tomancak P, Cardona A (2012) Fiji: an open-source platform for biological-image analysis. Nat Methods 9:676-682.

Schwarz LA, Miyamichi K, Gao XJ, Beier KT, Weissbourd B, DeLoach KE, Ren J, Ibanes S, Malenka RC, Kremer EJ, Luo L (2015) Viral-genetic tracing of the input-output organization of a central noradrenaline circuit. Nature 524:88-92.

Seagraves KM, Arthur BJ, Egnor SR (2016) Evidence for an audience effect in mice: male social partners alter the male vocal response to female cues. J Exp Biol 219:1437-1448.

Singewald G, Nguyen N, Neumann I, Singewald N, Reber S (2009) Effect of chronic psychosocial stress-induced by subordinate colony (CSC) housing on brain neuronal activity patterns in mice. Stress 12:58-69.

Sporns O (2010) Networks of the brain. Cambridge: MIT Press.

Tanimizu T, Kenney JW, Okano E, Kadoma K, Frankland PW, Kida S (2017) Functional connectivity of multiple brain regions required for the consolidation of social recognition memory. J Neurosci $37: 4103-4116$.

Toth M, Tulogdi A, Biro L, Soros P, Mikics E, Haller J (2012) The neural background of hyper-emotional aggression induced by postweaning social isolation. Behav Brain Res 233:120-129.

Tran L, Lasher BK, Young KA, Keele NB (2013) Depletion of serotonin in the basolateral amygdala elevates glutamate receptors and facilitates fear-potentiated startle. Transl Psychiatry 3:e298.

Tschida K, Michael V, Takatoh J, Han B-X, Zhao S, Sakurai K, Mooney R, Wang F (2019) A specialized neural circuit gates social vocalizations in the mouse. Neuron 103:459-472.e4. van den Heuvel MP, Sporns O (2019) A cross-disorder connectome landscape of brain dysconnectivity. Nat Rev Neurosci 20:435446.

Van den Heuvel MP, Bullmore ET, Sporns O (2016) Comparative connectomics. Trends Cogn Sci 20:345-361.

VanElzakker M, Fevurly RD, Breindel T, Spencer RL (2008) Environmental novelty is associated with a selective increase in Fos expression in the output elements of the hippocampal formation and the perirhinal cortex. Learn Mem 15:899-908.

Warren MR, Spurrier MS, Roth ED, Neunuebel JP (2018) Sex differences in vocal communication of freely interacting adult mice depend upon behavioral context. PLoS One 13:e0204527.

Warren M, Clein R, Spurrier M, Roth E, Neunuebel J (2020) Ultrashort-range, high-frequency communication by female mice shapes social interactions. Sci Rep 10:2637.

Watts DJ, Strogatz SH (1998) Collective dynamics of 'small-world' networks. Nature 393:440-442.

Wei YC, Wang SR, Jiao ZL, Zhang W, Lin JK, Li XY, Li SS, Zhang X, $\mathrm{Xu} \mathrm{XH} \mathrm{(2018)} \mathrm{Medial} \mathrm{preoptic} \mathrm{area} \mathrm{in} \mathrm{mice} \mathrm{is} \mathrm{capable} \mathrm{of} \mathrm{mediating}$ sexually dimorphic behaviors regardless of gender. Nat Commun 9:279.

Weitekamp CA, Hofmann HA (2017) Neuromolecular correlates of cooperation and conflict during territory defense in a cichlid fish. Horm Behav 89:145-156.

Williamson CM, Klein IS, Lee W, Curley JP (2019) Immediate early gene activation throughout the brain is associated with dynamic changes in social context. Soc Neurosci 14:253-265.

Wise T, Marwood L, Perkins AM, Herane-Vives A, Joules R, Lythgoe DJ, Luh W-M, Williams SCR, Young AH, Cleare AJ, Arnone D (2017) Instability of default mode network connectivity in major depression: a two-sample confirmation study. Transl Psychiatry 7: e1105. 